\title{
L'OSLÉROSE DU CHIEN : ACTUALITÉS DIAGNOSTIQUES ET THÉRAPEUTIQUES
}

\author{
BOURDOISEAU G *o et CADORÉ JL ** \\ avec la collaboration de FOURNIER C.* et GOUNEL J.M*
}

Summary : VERMINOUS BRONCHITIS IN THE DOG : UPDATE ON DIAGNOSIS AND TREATMENT

Oslerus osleri is a metastrongle whose first-stage larvae are expectorated by the bitch, and which are infecting for pups; few cases are described from France. The biological features of this parasite, diagnosis and therapy are described in a first bibliographic part. 29 of 66 dogs examined were infected with Oslerus osleri. We investigated the cause of their chronic coughing, which was not cured by routine therapies. Several diagnostic methods were used of which endoscopy was the best.

The following drugs were used to treat infection with Oslerus osleri : ivermectin, levamisole, oxfendazole and fenbendazole. The last-mentioned two drugs are safe for dogs and very effective against this parasite.

KEY WORDS : helminthiasis by Oslerus. Oslerus osleri. metastrongylide. diagnosis, treatment.

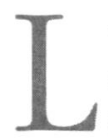

'oslérose est une helminthose respiratoire contagieuse et spécifique, affectant particulièrement le jeune chien, due à la migration dans l'organisme de larves, puis à l'installation, dans des nodules situés de part et d'autre du carrefour trachéobronchique, des adultes d'un strongle métastrongylidé Oslerus osleri (Cobbold, 1879). Ce parasite, décrit initialement à Montréal par Osler en 1877, a une très vaste répartition géographique (tableau I) : l'article de Mills, 1967 fournit une excellente synthèse en la matière (les références postérieures à cette publication sont citées dans la bibliographie); paradoxalement, peu de cas ont été décrits en France (tableau II).

C'est à partir d'élevages contaminés qu'il nous a été donné de suivre que nous nous proposons, après une revue bibliographique générale, d'envisager les particularités et difficultés diagnostiques et thérapeutiques inhérentes à la maladie.

Département des Sciences cliniques, École vétérinaire de Lyon, BP 83, 69280 MARCY

* Parasitologie et maladies parasitaires

** Pathologie médicale des Équidés et des Carnivores

- auteur auquel doit être adressée la correspondance; tél : 788725

73 ; télécopie : 78872614

\section{Résumé}

Oslerus osleri est un métastrongylidé dont la transmission est assurée par des larves de stade 1 expectorées par la chienne; peu de cas ont été décrits en France. Les caractères biologiques de ce parasite, le diagnostic et la thérapeutique de l'oslérose sont analysés dans une première partie bibliographique.

Sur 66 chiens examinés, 29 sont infestés. Les animaux sont présentés à la consultation pour une toux chronique rebelle aux thérapeutiques usuelles. Diverses méthodes diagnostiques sont utilisées parmi lesquelles il faut retenir l'endoscopie comme méthode de choix. Plusieurs molécules sont testées: l'ivermectine, le lévamisole, l'oxfendazole et le fenbendazole. Ces deux dernières molécules s'avèrent, dans de bonnes conditions de sécurité, très efficaces à l'encontre de ce parasite.

MOTS CLES : oslérose. Oslerus osleri. métastrongylidé, diagnostic, traitement.

\section{REVUE BIBLIOGRAPHIQUE}

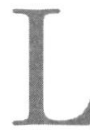

es tableaux I et II permettent de souligner les faits suivants :

- Oslerus osleri est un parasite de canidés : espèces sauvages (loup, coyote, dingo, renard), et chien domestique.

Foreyt \& Foreyt, 1981, grâce à un essai de transmission expérimentale du coyote au chien, démontrent que le canidé sauvage ne joue pas le rôle de réservoir pour le chien; la prévalence chez le coyote, d'après une enquête effectuée dans l'état de Washington, atteint $49 \%$. En outre, les rats, suspectés de jouer un rôle comme hôtes paraténiques ou hôtes de transport éventuels, ne semblent pas intervenir dans l'épidémiologie de l'oslérose. Dunsmore \& Spratt, 1979 confirment la transmission entre dingos, entre chiens, et l'absence d'infestation chez le chat.

- Ce métastrongylidé a été décrit dans de nombreux pays, avec une nette prédominance des pays anglosaxons (Etats-Unis, Angleterre, Australie,... : Mills, 1967), alors que des pays comme la France semblaient épargnés. Vraisemblablement, grâce à la connaissance que nous avons du cycle évolutif et de la transmission du parasite, l'incidence élevée récemment observée en France est la conséquence de chiens parasités importés dans des élevages. 


\begin{tabular}{|c|c|c|}
\hline PAYS & AUTEURS, ANNÉE & ESPËCE ANIMALE \\
\hline Canada & $\begin{array}{c}\text { Osler, } 1877 \\
\text { Ridell \& Henry, } 1966 \\
\text { Polley, } 1986\end{array}$ & $\begin{array}{l}\text { chien } \\
\text { loup } \\
\text { coyote }\end{array}$ \\
\hline USA & $\begin{array}{c}\text { Milks \& Muldoon,1915 } \\
\text { Price, } 1928 \\
\text { Erickson, } 1944 \\
\text { Pence \& Meinzer, } 1979\end{array}$ & $\begin{array}{l}\text { chien } \\
\text { coyote } \\
\text { loup, coyote } \\
\text { coyote }\end{array}$ \\
\hline Grande-Bretagne & $\begin{array}{c}\text { Pillers, } 1928,1935 \\
\text { Hare, } 1930 \\
\text { Ascoli, } 1936 \\
\text { Urquhart, } 1954\end{array}$ & $\begin{array}{c}\text { chien } \\
\text { chien } \\
\text { chien Cairn Terrier } \\
\text { chien Greyhound }\end{array}$ \\
\hline Allemagne & Rabe, 1883 & $\begin{array}{l}\text { chien Greyhound } \\
\text { importé d'Angleterre }\end{array}$ \\
\hline Australie & $\begin{array}{c}\text { Keep, } 1951 \\
\text { Dunsmore \& Spratt, } 1979\end{array}$ & $\begin{array}{c}\text { chien } \\
\text { dingo, renard }\end{array}$ \\
\hline Nouvelle-Zélande & Hopkirk, 1928 & chien Collie \\
\hline Afrique du Sud & $\begin{array}{c}\text { Ortlepp, } 1945 \\
\text { Steyn, 1945 } \\
\text { Malherbe, 1951, } 1954 \\
\text { Dorrington, 1959, } 1963\end{array}$ & $\begin{array}{c}\text { chien } \\
\text { chien } \\
\text { chien } \\
\text { chien Boxer, Bull Mastiff }\end{array}$ \\
\hline Inde & Gaiger, 1909 & chien Foxhound \\
\hline Russie & $\begin{array}{c}\text { Blumberg, } 1882 \\
\text { Doroschenko, } 1906\end{array}$ & $\begin{array}{l}\text { chien } \\
\text { chien }\end{array}$ \\
\hline
\end{tabular}

Tableau I. - Répartition géographique de l'oslérose à partir des cas cliniques décrits dans la littérature.

\begin{tabular}{|c|c|}
\hline AUTEURS & ANNÉE \\
\hline Renaud (in Neumann,1941) & 1835 \\
Courtin (id) & 1882 \\
Megnin (id) & 1883 \\
Cotard \& Bourdeau & 1982 \\
Vallet-Picavet & 1991 \\
Bourdoiseau \& Cadoré & 1993 \\
\hline
\end{tabular}

Tableau II. - Descriptions en France portant uniquement sur des chiens.

Diverses enquêtes ont montré une prévalence élevée chez le chien en Angleterre : jusqu'à 18,5\% d'après Jacobs \& Prole, 1976, chez les chiens de race Greyhound, $18 \%$ chez les chiens de même race et $6,25 \%$ pour d'autres races d'après Hovel \& Weston, 1970 ; ces résultats sont toutefois discutables, dans la mesure où ils sont obtenus avec des méthodes diagnostiques variées, dont certaines (comme la coproscopie) reconnues pour leur faible sensibilité.

Aucune enquête de ce type n'a été réalisée en France : la prévalence n'y est donc pas connue ; en revanche, l'incidence est certainement élevée car, depuis les premiers cas cliniques décrits en 1982, il n'est pas rare désormais d'observer cette helminthose au sein d'élevages, en particulier de caniches, même si cette race n'est pas plus prédisposée que les autres.

\section{LE PARASITE : OsLERUS OSLERI (COBBOLD, 1879)}

Oslerus osleri (parfois désigné sous le binôme Filaroides osleri) est un nématode métastrongylidé ou "strongle respiratoire".

L'adulte mâle mesure $5 \mathrm{~mm}$ de longueur, est pourvu d'une bourse caudale atrophiée munie de deux spicules de $100 \mu \mathrm{m}$, légèrement incurvés. L'adulte femelle est plus long (10 à $15 \mathrm{~mm}$ ), présente une cavité buccale réduite, suivie d'un oesophage dont l'appareil valvulaire est difficilement visible.

Les oufs sont ovales $(90 \mu \mathrm{m}$ de longueur sur $60 \mu \mathrm{m}$ de largeur), munis d'une paroi mince, renfermant une larve. Les larves de stade 1 mesurent 230 à $260 \mu \mathrm{m}$ de longueur, pourvues d'un œesophage long (le tiers de la longueur totale) et rhabditoïde; l'extrémité distale est caractéristique, incurvée en S (Euzeby, 1961; Bourdeau, 1992).

Ce strongle respiratoire, non hématophage, est logé dans des nodules intéressant la muqueuse et la sousmuqueuse respiratoires, situés de part et d'autre du carrefour trachéobronchique; ces nodules varient dans leur aspect (plus ou moins opaques, jaunâtres, ou au contraire transparents, clairs) et dans leurs dimensions (quelques $\mathrm{mm}$ à $1 \mathrm{~cm}$ de longueur). L'examen endoscopique permet de bien voir les parasites par transparence dans ces nodules. L'extrémité postérieure de la femelle est en communication avec 
la lumière trachéale, permettant ainsi la libération des larves de stade 1 dans l'arbre aérifère.

Le cycle évolutif est resté pendant longtemps mystérieux : Osler, 1877 avance l'hypothèse d'une transmission par inhalation des embryons, Urquhart et al., 1954 celle d'un cycle direct; Ortlepp, 1945, par analogie avec ce que l'on sait à propos des autres métastrongylidés (en particulier des espèces voisines du genre Filaroides), pense qu'un hôte intermédiaire (escargot ou limace) intervient dans le cycle évolutif.

C'est Dorrington, 1968 qui, par de brillantes expériences, définit le stade infestant (la larve de stade 1), les modalités de la transmission et les étapes successives de la migration dans l'organisme (fig. 1) :

- des chiots issus de mères infestées, séparés d'elles dès la naissance et élevés par des chiennes saines restent indemnes : la transmission in utero n'existe pas;

- l'administration plusieurs jours de suite, par voie orale, d'œufs de Oslerus osleri recueillis à partir d'une chienne infestée assure la transmission aux chiots; il n'y a pas d'hôte intermédiaire;

- aucune prédisposition de sexe n'est observée, mais le chien reste susceptible quel que soit son âge; la forte prévalence de l'infestation observée chez le jeune chien tient à son comportement et à son intimité avec la chienne;

- en effet, aucune transmission entre chiots n'est démontrée : c'est la mère qui, par la toilette et les soins qu'elle prodigue à sa portée, est responsable directement de la contamination des chiots, par les larves de stade 1 expectorées à la faveur de la toux ; ces larves, dans le milieu extérieur, sont très sensibles à la dessiccation et ne résistent que quelques heures;

- les chiots déglutissent les larves qui atteignent rapidement l'estomac, puis l'intestin grêle pour gagner les voies lymphatiques et le ganglion mésentérique (12h après leur ingestion) où s'effectue la première mue pour donner des larves de stade 2; celles-ci arrivent, via le canal thoracique pour la plupart d'entre elles, via la veine porte, le foie puis la veine cave caudale pour une minorité, dans le coeur droit; les larves atteignent les poumons où a lieu la deuxième mue qui donne des larves de stade 3, celles-ci remontent l'arbre aérifère pour atteindre la bifurcation trachéale; les localisations des mues 3 et 4 pour donner des larves 4 et des juvéniles ne sont pas connues.

- la période prépatente est de 10 semaines; la période patente n'est pas connue avec précision, mais la chienne est susceptible d'expectorer, de façon intermittente, des larves infestantes durant plusieurs années.

\section{ÉPIDÉMIOLOGIE ET CLINIQUE}

Les caractères épidémiologiques et cliniques propres à cette helminthose sont directement issus des modalités particulières du cycle évolutif et de la transmission du parasite (Clayton \& Lindsay, 1979). L'oslérose est une helminthose se déclarant essentiellement :

- chez des chiens élevés en groupe : il s'agit d'une helminthose observée en chenil, même si des cas peuvent être observés sur des animaux isolés, qui sont alors des animaux récemment acquis et issus d'une collectivité infestée;

- chez des chiens de toute race : les descriptions initiales ayant été faites par des auteurs anglosaxons, les premiers cas ont été décrits chez des races élevées en ces pays : Greyhound, Cavalier King Charles, chien de Poméranie, Jack Russell Terrier, Cairn Terrier, Boxer, Beagle, Bull Mastiff, Cocker Spaniel, Labrador, etc.(Brownlie 1990, Dorrington 1968, Jacobs \& Prole 1976). En France, c'est le caniche qui semble être fréquemment infesté, les animaux appartenant à cette race ayant introduit l'oslérose dans notre pays;

- chez des chiens jeunes : l'infestation a lieu dans les premières semaines de la vie lorsque le chiot est sous la mère; la période prépatente étant de 10 semaines,

Chienne (période patente : plusieurs années)

adultes présents dans des nodules situés de part et d'autre de la bifurcation bronchique larves de stade 1 immédiatement infestantes expectorées

$\begin{array}{lll}\begin{array}{l}\text { parasites } \\ \text { adultes remontent } \\ \text { l'arbre aérifère } \\ \text { (mues 3 et 4) }\end{array} & \begin{array}{l}\text { elles } \\ \text { coeur droit et } \\ \text { aux poumons } \\ \text { (mue 2) }\end{array}\end{array}$

chiot (période prépatente : 10 semaines)

Fig. 1. - Schéma du cycle évolutif de Oslerus osleri 
la maladie se déclare chez des chiots âgés au minimum de 2 mois et demi, c'est à dire au moment du sevrage et de la vente des animaux; toutefois, un chien reste réceptif toute sa vie: un chien adulte mis en pension dans un élevage contaminé pourra contracter l'oslérose.

Ainsi, l'oslérose est une helminthose contagieuse, du jeune chien, sévissant en collectivité.

D'un point de vue clinique, l'oslérose se traduit par une toux sèche, quinteuse, chronique et non hyperthermisante : elle peut être confondue avec le syndrome de "toux de chenil", elle n'est généralement pas suivie de complications ni de dégradation de l'état général (rares cas de détresse respiratoire aiguë mortelle ou de pneumothorax : Burrows et al., 1972). Cette trachéobronchite ne rétrocède pas aux traitements antibiotiques et anti-inflammatoires classiques. (Barr et al., 1986; Bourdeau, 1982; Bourdoiseau \& Cadoré, 1993; Cotard, 1982; Dorrington, 1968; Pillers, 1935; Valet-Picavet, 1991; Wheeldon et al., 1977).

Le diagnostic de l'oslérose s'avère difficile : la clinique ne permet pas de différencier cette helminthose des autres causes de toux chronique du jeune chien (Bourdeau, 1982; Bourdoiseau \& Cadoré, 1993; Dorrington, 1968; Jacobs \& Prole, 1976); il est nécessaire de recourir à des investigations complémentaires:

- la coproscopie est aléatoire dans ses résultats du fait de l'élimination irrégulière des larves; Dorrington, 1968 utilise la technique de flottation et considère que cette méthode n'est pas fiable; même la technique de Baermann, pourtant plus sensible pour la mise en évidence des larves dans les fèces, s'avère décevante : Jacobs \& Prole 1976 montrent que cet examen est négatif alors que l'autopsie révèle 18,5\% de chiens parasités sur un effectif de 27 animaux examinés.

- l'examen du mucus respiratoire et des expectorations s'avère, selon Dorrington 1968, plus sensible que la coproscopie, mais ne peut être réalisé dans la pratique courante (Jones et al., 1977);

- la radiographie peut révéler, de façon inconstante, des opacités nodulaires dans la lumière trachéale, difficilement visibles au carrefour trachéobronchique; la lecture est difficile et cet examen peut avoir une valeur d'orientation diagnostique (Lauder \& Lawson, 1959);

- l'endoscopie trachéale est le seul examen fiable permettant de visualiser les nodules caractéristiques, d'effectuer une biopsie et ainsi de confirmer la présence du parasite (Pillers, 1935; Dorrington, 1968).

La thérapeutique est décevante : peu d'anthelminthiques sont efficaces à l'encontre de ces nématodes non hématophages, situés dans et sous la muqueuse respiratoire (Oakley \& Rowlands, 1991). Ils sont présentés dans le tableau III.

\section{DIFFICULTÉS DIAGNOSTIQUES. INTÉRÊT DE L'ENDOSCOPIE.}

\author{
MATÉRIEl ET MÉTHODE.
}

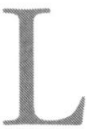

a consultation d'animaux à l'École vétérinaire de Lyon, pour un motif de toux chronique, nous a amené à établir, pour plusieurs d'entre eux, un diagnostic d'oslérose : les investigations complémentaires nécessaires et l'examen systématique des animaux des élevages contaminés d'origine sont exposés.

Animaux (tableau IV)

Les observations cliniques ont toutes été effectuées sur des chiens de race Caniche .Ces animaux peuvent être divisés, selon leur origine, en quatre catégories : chiens d’âges variés, issus de trois élevages; jeunes chiens, récemment acquis par leur nouveau propriétaire à partir des élevages précédents.

En outre, la fréquence élevée des cas cliniques chez les chiots et les jeunes chiens nous a incité à diviser les animaux en deux classes d'âge : une classe regroupant les animaux de moins de un an, et une pour les animaux de plus de un an.

Les 66 animaux examinés à l'École Vétérinaire pour un motif de toux chronique l'ont été sur une période de 18 mois environ.

Matériel

Le matériel utilisé est le suivant : matériel de laboratoire nécessaire à la réalisation d'une coproscopie par flottation à l'iodomercurate de potassium (solution préparée à partir de bi-iodure de mercure et d'iodure de potassium, Comptoir Lyonnais de Verrerie, Villeurbanne) reconstitué en notre laboratoire (Euzeby, 1981); matériel radiographique classique de l'école (appareil Siemens Pandows Optimatic $\left.\mathrm{n}^{\circ} 1416\right)$; endoscope de marque Olympus GIF-XP10, pour la trachéoscopie de chiens de petite taille (Cadoré, 1992; Lecoindre \& Cadoré, 1992 ; Lamure-Sauvaire, 1992).

Méthode

Tous les animaux sont examinés, puis tranquillisés (Valium (B) : $0,25 \mathrm{mg} / \mathrm{kg}$ par la voie intra-musculaire) et anesthésiés (Imalgène 500 ß : $5 \mathrm{mg} / \mathrm{kg}$ par la voie intraveineuse) en vue de l'examen endoscopique. Quand cela a été possible, une radiographie de profil a été réalisée ainsi qu'une coproscopie.

\section{RÉSULTATS}

Sur les 66 animaux examinés, ont été considérés comme infestés les animaux positifs à l'une au moins des trois investigations complémentaires réalisées : la coproscopie, la radiographie et l'endoscopie. Les résultats sont exposés dans les tableaux V, VI et VII. 


\begin{tabular}{|c|c|c|}
\hline MOLÉCULE & UTILISATION & RÉFÉRENCE \\
\hline lévamisole & $7,5 \mathrm{mg} / \mathrm{kg} / \mathrm{j}$ p.o ou s.c $\times 10-30 \mathrm{j}$ & $\begin{array}{c}\text { Darke, } 1976 \\
\text { Randolph \& } \\
\text { Rondano, } 1984\end{array}$ \\
\hline thiabendazole & $\begin{array}{l}64 \mathrm{mg} / \mathrm{kg} / \mathrm{j} \text { p.o x } 21 \mathrm{j} \\
64 \mathrm{mg} / \mathrm{kg} / \mathrm{j} \text { p.o } \times 21 \mathrm{j}\end{array}$ & $\begin{array}{c}\text { Bennett, } 1975 \\
\text { Bennett \& } \\
\text { Beresford-Jones, } 1973\end{array}$ \\
\hline albendazole & $9,5 \mathrm{mg} / \mathrm{kg} / \mathrm{j}$ p.o $\times 55 \mathrm{j}$ & $\begin{array}{c}\text { Van Heerden \& } \\
\text { Petrick, 1980, } \\
\text { in Kelly \& Mason }\end{array}$ \\
\hline oxfendazole & $10 \mathrm{mg} / \mathrm{kg} / \mathrm{j}$ p.o $\times 28 \mathrm{j}$ & Kelly \& Mason, 1985 \\
\hline ivermectine & $0,4 \mathrm{mg} / \mathrm{kg} \mathrm{s} . \mathrm{C}$ & $\begin{array}{c}\text { Clayton, } 1983 \\
\text { in Kelly \& Mason }\end{array}$ \\
\hline
\end{tabular}

Tableau III. - Anthelminthiques préconisés dans la littérature à l'encontre de Oslerus osleri. p.o. : par la voie orale - s.c : par la voie sous-cutanée

\begin{tabular}{|c|c|c|c|c|c|c|c|c|c|c|c|c|c|}
\hline \multirow{2}{*}{$\begin{array}{l}\text { classe } \\
\text { d'âge }\end{array}$} & \multicolumn{4}{|c|}{$\begin{array}{c}\text { origine des chiens : } \\
4 \text { catégories }\end{array}$} & \multicolumn{2}{|c|}{ sexe } & \multicolumn{2}{|c|}{$\begin{array}{c}\text { motif }^{1} \text { de } \\
\text { consultation }\end{array}$} & \multicolumn{4}{|c|}{$\begin{array}{l}\text { examen clinique } \\
\text { des animaux }\end{array}$} & \multirow[t]{2}{*}{ total } \\
\hline & $\begin{array}{l}\text { élev. } \\
n^{\circ} 1\end{array}$ & $\begin{array}{l}\text { élev. } \\
n^{\circ} 2\end{array}$ & $\begin{array}{l}\text { élev. } \\
\mathrm{n}^{\circ} 3\end{array}$ & $\begin{array}{l}\text { cas } \\
\text { isolé }\end{array}$ & fem. & mâle & + & - & 0 & + & ++ & +++ & \\
\hline$<1$ an & 1 & 4 & 1 & 5 & 6 & 5 & 8 & 3 & 3 & 3 & 3 & 2 & 11 \\
\hline$>1$ an & 34 & 14 & 6 & 1 & 36 & 19 & 10 & 45 & 45 & 9 & 1 & 0 & 55 \\
\hline total & 35 & 18 & 7 & 6 & 42 & 24 & 18 & 48 & 48 & 12 & 4 & 2 & 66 \\
\hline
\end{tabular}

Tableau IV. - Caractéristiques des animaux examinés.

1. motif de consultation : soit l'animal présente l'un quelconque des symptômes de l'oslérose, et il est considéré comme suspect : +; soit l'animal ne présente aucun symptôme évocateur :-

2. examen clinique des animaux : selon la nature et l'intensité des symptômes, 4 groupes d'animaux sont définis : animaux en parfait état général : 0 (et il s'agit des même animaux que ceux rangés sous la rubrique "motif -"; animaux en bon état général, mais souffrant de toux : +; animaux souffrant de toux importante et de dyspnée : ++; animaux présentant toux, dyspnée et dégradation de l'état général : +++ (ces trois derniers groupes constituant l'ensemble des sujets regroupés sous la rubrique "motif +").

\begin{tabular}{|c|c|c|c|c|c|c|c|c|c|c|}
\hline \multirow{2}{*}{ motif } & \multirow{2}{*}{$\begin{array}{l}\text { examen } \\
\text { clinique }\end{array}$} & \multicolumn{3}{|c|}{ examens coproscopiques } & \multicolumn{3}{|c|}{ examens radiographiques } & \multicolumn{3}{|c|}{ examens endoscopiques } \\
\hline & & réalisés & + & - & réalisés & + & - & réalisés & + & - \\
\hline$-: 48$ & $0: 48$ & 1 & 1 & 0 & 1 & 0 & 1 & 48 & 11 & 37 \\
\hline \multirow{3}{*}{$+: 18$} & $+: 12$ & 6 & 6 & 0 & 5 & 4 & 1 & 12 & 12 & 0 \\
\hline & $++: 4$ & 3 & 2 & 1 & 2 & 1 & 1 & 4 & 4 & 0 \\
\hline & $+++: 2$ & 2 & 0 & 2 & 2 & 1 & 1 & 2 & 2 & 0 \\
\hline total & 66 & 12 & 9 & 3 & 10 & 6 & 4 & 66 & 29 & 37 \\
\hline
\end{tabular}

Tableau V. - Résultats des investigations complémentaires.

\begin{tabular}{|c|c|c|}
\hline classes d'âge des animaux & effectif d'animaux examinés : 66 & effectif d'animaux parasités : 29 \\
\hline animaux $<1$ an & 11 & 8 \\
\hline animaux $>1$ an & 55 & 21 \\
\hline \% d'animaux de moins de 1 an & $11 / 66=16,66 \%$ & $8 / 29=27,58 \%$ \\
\hline
\end{tabular}

Tableau VI. - Proportions relatives des animaux examinés et parasités en fonction des classes d'âge.

\begin{tabular}{|c|c|c|c|}
\hline \multicolumn{2}{|c|}{$\begin{array}{c}\text { animaux parasités } \\
\text { (c'est à dire positifs à un examen } \\
\text { complémentaire au moins) }=29\end{array}$} & nombre respectif de faux négatifs \\
\hline coproscopie & 12 réalisées & 9 positives & 3 animaux négatifs se sont révélés positifs en endoscopie \\
\hline radiographie & 10 réalisées & 6 positives & \begin{tabular}{l}
4 animaux négatifs se sont révélés positifs en endoscopie \\
\hline endoscopie
\end{tabular} \\
\hline 66 réalisées & 29 positives & $\begin{array}{l}\text { aucun animal négatif en endoscopie ne s'est révélé positif } \\
\text { par une autre méthode }\end{array}$ \\
\hline
\end{tabular}

Tableau VII. - Proportions relatives des faux négatifs en fonction de chaque méthode diagnostique. 


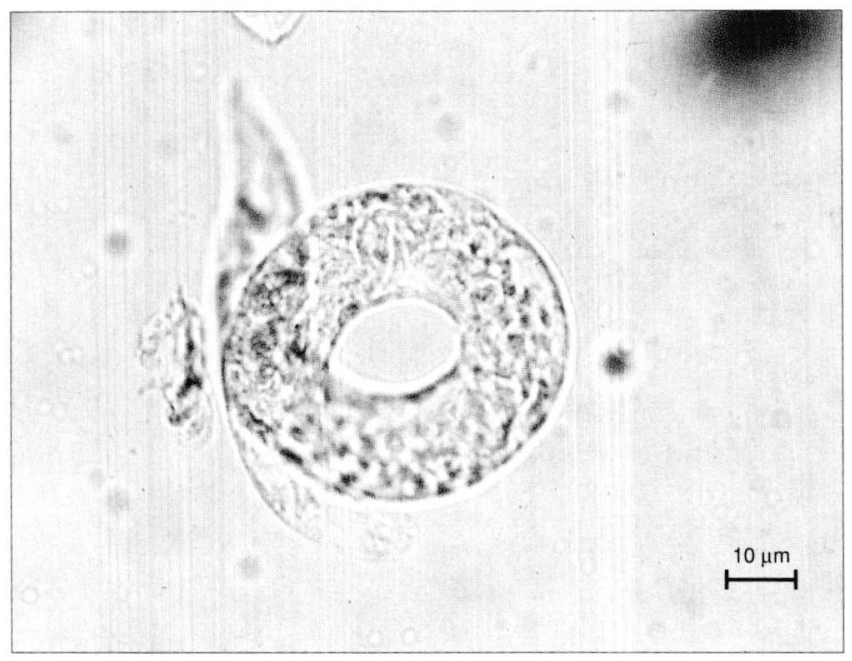

Fig. 2. - Larve de Oslerus osleri de stade 1 observée par coproscopie.

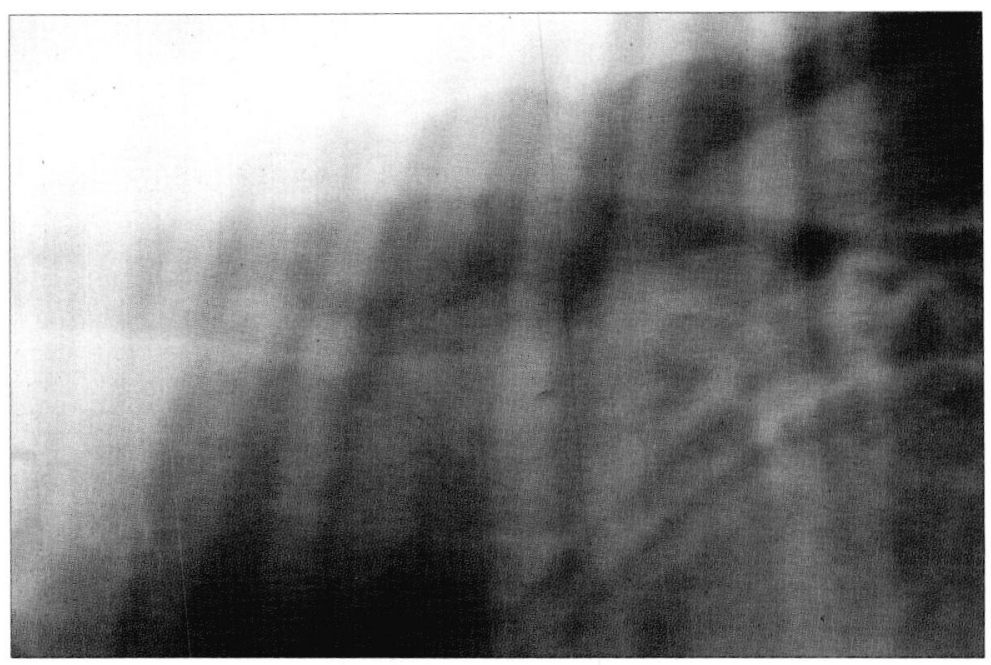

Fig. 3. - Radiographie de profil d'un thorax de chien infesté : noter les petites ponctuations opaques dans la lumière trachéale.

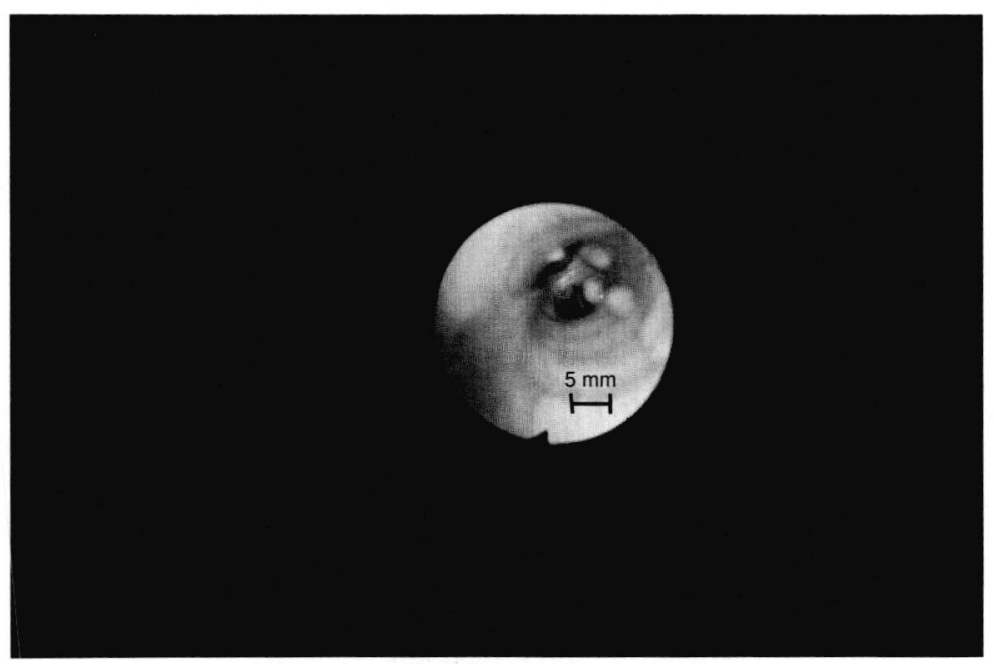

Fig. 4. - Nodules visibles par endoscopie à la bifurcation trachéale.

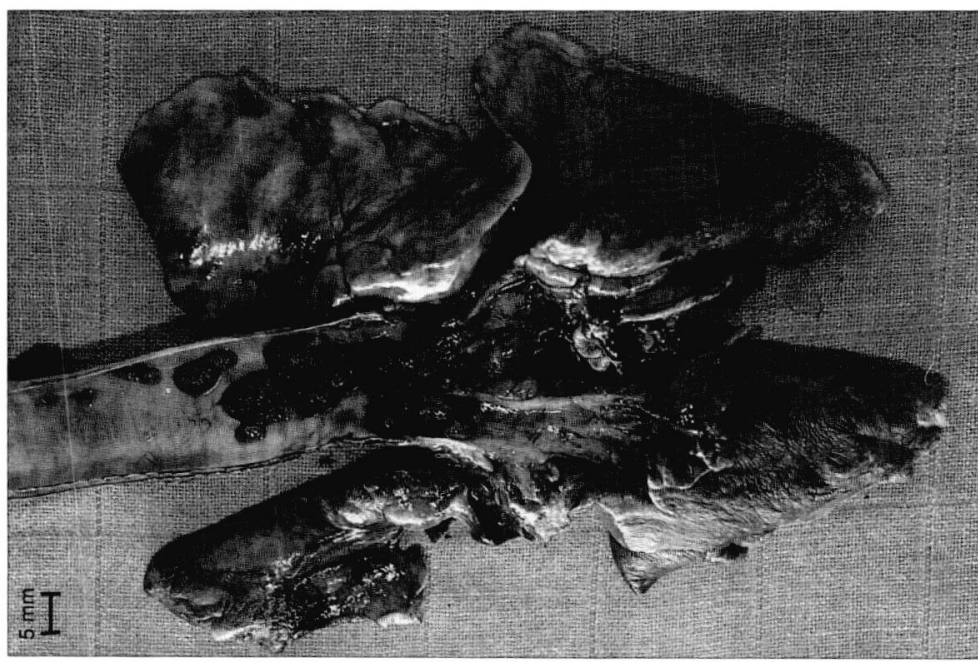

Fig. 5. - Nodules envahissant la lumière trachéale, à l'autopsie d'un jeune chien mort d'oslérose. 


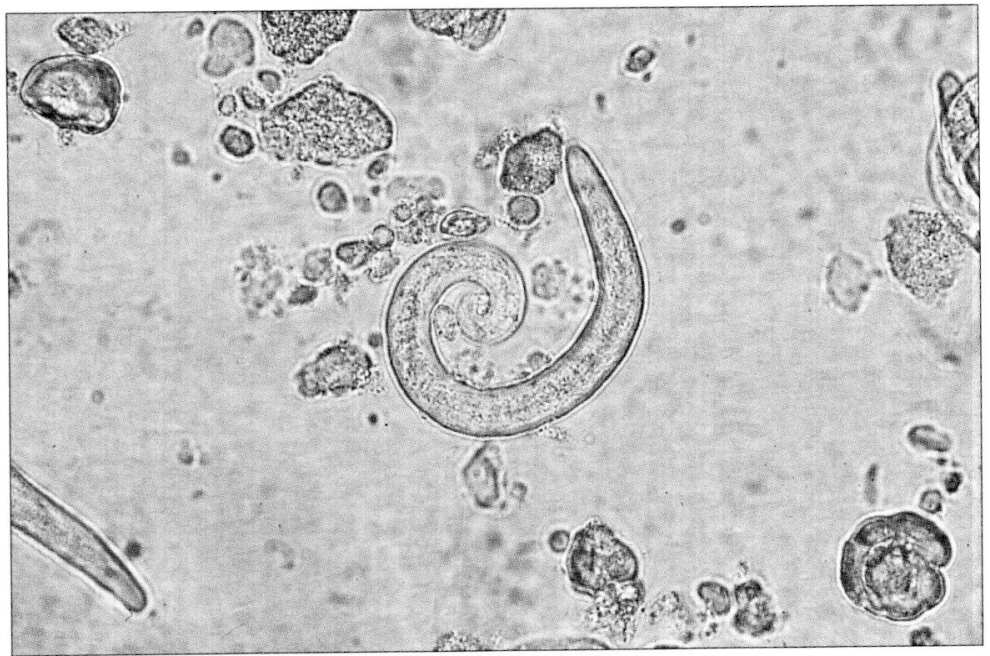

Fig. 6. - Extrémités antérieure (à gauche) et postérieure (à droite) d'une femelle de Oslerus osleri.

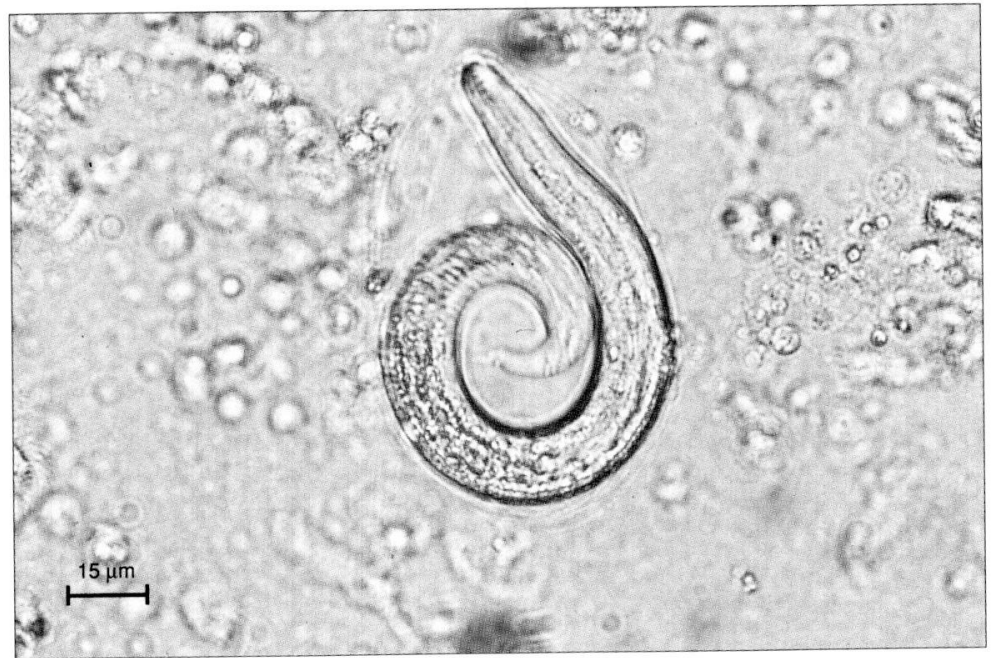

Fig. 7. - CEuf larvé recueilli à la faveur d'une biopsie d'un nodule trachéal à la faveur d'une endoscopie.

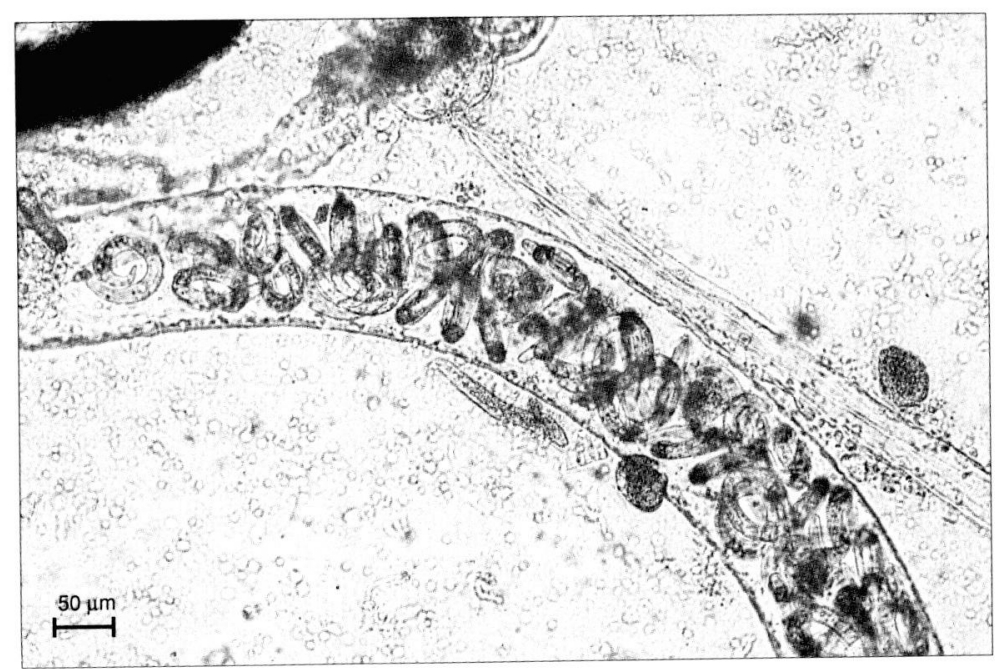

Fig. 8. - Femelle gravide recueillie à la faveur d'une biopsie. 


\section{Discussion}

Quant à l'effectif des animaux examinés, le tableau IV mérite les commentaires suivants :

- sur un effectif total de 66 chiens examinés, 60 appartiennent à des éleveurs : seuls, en effet, les élevages étaient en mesure de nous fournir un effectif important de chiens ayant été en contact avec des animaux infestés;

- pour cette raison également, on observe un déséquilibre dans le sex-ratio des animaux examinés, les éleveurs gardant plus de femelles (la plupart âgées de plus de deux ans car destinées à la reproduction) que de mâles;

- les cas isolés sont de jeunes animaux : cinq ont moins de un an, un seul a plus de deux ans; ceci confirme le caractère épidémiologique essentiel de l'oslérose : les symptômes sont observés dans les premiers mois de la vie.

Concernant le diagnostic, (cf. tableaux V et VI) l'étude clinique que nous avons pu mener confirme les caractères de l'oslérose : cette helminthose apparaît classiquement comme une trachéobronchite chronique, apyrétique, le plus souvent sans atteinte de l'état général, sévissant chez de jeunes animaux, dans les semaines qui suivent leur achat. La plupart du temps, ces jeunes chiens sont traités pour une toux de chenil ou une bronchite infectieuse classique; le caractère récidivant de la toux chez un jeune animal récemment acheté dans un chenil constitue un élément important de suspicion, les traitements antibiotiques associés aux corticoïdes et aux antitussifs ne produisant qu'une amélioration passagère. Le tableau VI traduit bien la fréquence particulièrement élevée de l'oslérose chez les jeunes animaux.

La méthode diagnostique de choix est l'endoscopie: la coproscopie (fig. 2) et la radiographie (fig. 3) sont entachées d'un taux de faux négatifs trop élevé (cf. tableau VII); elles peuvent constituer un appoint intéressant dans le cadre de la première consultation motivée par la toux, parce que d'exécution et de lecture rapides : ces techniques permettent donc, quand le résultat est positif, d'établir un diagnostic immédiat; en revanche, lors de consultation motivée par une toux récidivante, ces méthodes doivent être écartées au profit de l'endoscopie (fig. 4).

\section{DIFFICULTÉS THÉRAPEUTIQUES. INTÉRÊT DES BENZIMIDAZOLES}

\section{MATÉRIEL ET MÉTHODE}

es animaux parasités définis dans le tableau IV ont été traités selon l'un des protocoles suivants :
- injection par la voie sous-cutanée d'ivermectine (Ivomec ${ }^{\circledR}$ bovin solution injectable) à la posologie de $0,2 \mathrm{mg} / \mathrm{kg}$; deux injections sont pratiquées à deux semaines d'intervalle;

- administration par la voie orale du lévamisole à la dose de $7,5 \mathrm{mg} / \mathrm{kg}$, tous les jours pendant trois semaines;

- administration d'ivermectine in situ, (Ivomec ${ }^{\circledR}$ bovin solution injectable) dans le nodule observé à l'endoscope;

- administration par la voie orale de fenbendazole (Panacur 10\% ®) à la dose de $50 \mathrm{mg} / \mathrm{kg} / \mathrm{j}$ pendant 15 jours;

- administration par la voie orale d'oxfendazole (Dolthène ${ }^{\circledR}$ ) à la dose de $50 \mathrm{mg} / \mathrm{kg} / \mathrm{j}$ pendant 15 jours.

Les animaux sont systématiquement examinés et contrôlés par une endoscopie au terme de leur traitement : dans le cas d'observation de nodules, ceux-ci sont prélevés, leur contenu observé au microscope.

\section{RÉSULTATS}

Les résultats des diverses thérapeutiques sont exposés dans le tableau VIII :

- sont considérés comme infestés les animaux présentant encore, à l'endoscopie, des "nodules actifs", c'est à dire dont la paroi est transparente et fine; l'aspect de ces nodules est caractéristique et se distingue bien à l'endoscope, avec un peu d'habitude, de nodules dégénérés ou fibreux; la biopsie confirme le caractère vivant du nodule en révélant, sous microscope, une femelle gravide de Oslerus osleri. (figs. 6, 7 et 8).

- sont considérés comme guéris les animaux ne présentant aucun nodule ou seulement des nodules fibreux, dont le contenu étalé au microscope s'avère dépourvu de tout matériel parasitaire.

Le total des animaux traités est supérieur au nombre d'animaux examinés car plusieurs chiens, du fait de l'échec d'une première thérapeutique, en ont reçu une seconde.

\section{DISCUSSION}

Le choix des molécules a été fondé sur les critères suivants :

- pour l'ivermectine : sa facilité d'utilisation et son large spectre font que, malgré son absence d'AMM dans l'espèce canine et ses risques toxiques, cette molécule présente un grand intérêt, en particulier au sein de grands effectifs;

- pour les benzimidazoles : leur spectre et leur fort index thérapeutique. Ces médicaments sont utilisés couramment chez le chien : seul le fenbendazole n'a pas d'autorisation d'utilisation en France. Ils sont 


\begin{tabular}{|c|c|c|c|}
\hline Thérapeutique pratiquée & animaux traités & animaux guéris & nombre d'échecs \\
\hline ivermectine voie sous-cutanée & 10 & 5 & 5 \\
\hline ivermectine in situ & 2 & 2 & 0 \\
\hline lévamisole per os & 1 & 0 & 1 \\
\hline fenbendazole per os & 19 & 19 & 0 \\
\hline oxfendazole per os & 4 & 4 & 0 \\
\hline total & 36 & 30 & 6 \\
\hline
\end{tabular}

Tableau VIII. - Résultats des thérapeutiques

remarquablement efficaces à l'encontre des nématodes, en particulier contre les larves en migration et les strongles respiratoires.

L'ivermectine s'est révélée décevante :

- plusieurs animaux traités ont présenté, dans les jours suivant la première (ou la seconde) injection, une amélioration clinique (disparition de la toux en particulier) associée à une négativation de la coproscopie; toutefois, la moitié de ces animaux a été représentée en consultation quelques semaines plus tard pour une rechute : l'endoscopie s'est révélée positive.

- l'injection in situ d'ivermectine ne peut être retenue, du fait de la difficulté technique, comme méthode thérapeutique usuelle.

L'ivermectine semble stériliser les animaux parasités (dans une proportion de $50 \%$ ) sans les guérir : la moitié des chiens ainsi traités éliminent de nouveau des larves infestantes. Bien que pratiquée sur deux animaux seulement, l'injection in situ démontre que la faible efficacité de l'ivermectine est due essentiellement à sa faible élimination dans l'appareil respiratoire. L'ivermectine, outre le risque de toxicité chez un certain nombre de races, ne peut être retenue du fait de résultats inconstants.

Le lévamisole est considéré par certains auteurs (tableau III) comme efficace sur Oslerus osleri : la durée du traitement associée au faible index thérapeutique de la molécule (de l'ordre de 2) nous ont poussé à ne pas retenir cette molécule comme thérapeutique de choix. Un chien a été traité de cette manière et s'est révélé parasité au contrôle endoscopique.

Le fenbendazole et l'oxfendazole, en revanche, associent efficacité et sécurité : toutes les endoscopies pratiquées chez les animaux traités se sont révélées négatives au terme du traitement, sans aucune rechute. Ces molécules possèdent un index thérapeutique élevé, comme tous les benzimidazoles, et sont donc utilisables chez les jeunes, les sujets souffrant d'insuffisance respiratoire et même, chez les femelles dans le dernier tiers de leur gestation.

Le fenbendazole toutefois, à la différence de l'oxfendazole, n'a pas d'autorisation officielle d'utilisation chez le chien en France, alors qu'il est utilisé couram- ment, chez cette espèce, dans les pays anglosaxons. Pour cette raison, et pour cette raison seulement, nous préférons actuellement retenir, au sein des benzimidazoles, l'oxfendazole pour traiter les animaux parasités. Ce résultat confirme ceux obtenus par Kelly \& Mason, 1985.

L'observation de chiens parasités par Oslerus osleri nous a permis d'établir les faits suivants :

- l'endoscopie constitue la méthode diagnostique de choix; en outre, elle permet de vérifier l'efficacité du traitement : la simple observation de la lumière trachéale doit être associée à la biopsie des nodules présents afin d'en vérifier le contenu ;

-la thérapeutique fait appel à l'oxfendazole (ou au fenbendazole sous la responsabilité du vétérinaire);

- la prophylaxie doit concerner l'introduction de chiens (en particulier les chiennes destinées à la reproduction) dans les élevages afin de contrôler efficacement cette helminthose à incidence probablement élevée dans notre pays.

\section{REMERCIEMENTS}

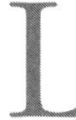

es auteurs remercient les professeurs Gevrey $\mathrm{J}$. et Chauve C. du laboratoire de parasitologie de l'École vétérinaire de Lyon pour leur lecture critique de ce manuscrit. Ils remercient également le laboratoire Distrivet pour avoir mis gracieusement à leur disposition le fenbendazole (Panacur $10 \%$ ( ) ainsi que Rhône Mérieux pour l'oxfendazole (Dolthène (®). Les photographies ont pu être réalisées en quadrichromie grâce à l'aide du laboratoire Rhône Mérieux.

\section{RÉFÉRENCES}

Ascoli E.W. Some experiences of parasitic tracheobronchitis in the dog due to Oslerus osleri. Veterinary Record, 1936, 16, 14-16.

Barr S.C., Lavelle R.B., Harrigan K.E. and Arundel J.H. Oslerus (Filaroides) osleri in a dog. Australian Veterinary Journal, 1986, 63, 334-337.

Bennetr D. The diagnosis and treatment of Filaroides osleri in the dog. Veterinary annual, 1975, 15, 256-260. 
Bennett D. and Beresford-Jones W.P. Treatment of Filaroides osleri infection in a 16 month old Yorkshire Terrier with thiabendazole. Veterinary Record, 1973, 93 , 226-227.

BOURdeAu P. La filaroïdose canine à Oslerus osleri (Filaroides osleri), données actuelles. Point vétérinaire, 1982, 14, 13-19.

Bourdeau P. Helminthoses respiratoires des carnivores. Encyclopédie Vétérinaire, 1992, Parasitologie, 0800, 9p.

Bourdoiseau G. and CAdoré J.L. Helminthoses respiratoires des carnivores domestiques. Recueil de Médecine Vétérinaire, 1993, 169, 415-420.

Brownlie S.E. A retrospective study of diagnosis in 109 cases of canine lower respiratory disease. Journal of Small Animal Practice, 1990, 31, 371-376.

Burrows C.F., O'Brifn J.A. and Biery D.N. Pneumothorax due to Filaroides osleri infestation in the dog. Journal of Small Animals Practice, 1972, 3, 613-618.

CADORÉ J.L. Endoscopie trachéo-bronchique chez le chien. Recueil de Médecine Vétérinaire, 1992, 168, 187-191.

Clayton H.M. and Lindsay F.E.F. Filaroides osleri infection in the dog. Journal of Small Animal Practice, 1979, 20, 773-782.

CoBbold T.S. Parasites. A treatise on the Entozoa of man and animals, including some account of the Cetozoa. J. and A. Churchill, 1879, London, 304 pp.

Cotard J.P. and Bourdeau P. Cas clinique : filaroïdose canine à Oslerus osleri. Point vétérinaire, 1982, 14, 8-10.

DARKe P.G.G. Use of levamisole in the treatment of parasitic tracheobronchitis in the dog. Veterinary Record, 1976, 99, 293-294.

DORRINGTON J.E. Studies of Filaroides osleri infestation in dogs. Onderstepoort Journal of Veterinary Research, 1968, 35, 225-286.

Dunsmore J.D. and SPRATT D.M. The life history of Filaroides osleri in wild and domestic canids in Australia. Veterinary Parasitology, 1979, 5, 275-286.

Euzeby J. Les maladies vermineuses des animaux domestiques et leurs incidences sur la pathologie humaine. Tome I : Maladies dues aux némathelminthes. Fascicule 1, Paris, Vigot, 1961, 473 pp.

Euzeby J. Diagnostic expérimental des helminthoses animales. Tome I. Informations techniques des Services Vétérinaires, Paris, 1981, 340 pp.

Foreyt W.J. and Foreyt K.M. Attempted transmission of Oslerus (Oslerus) osleri (= Filaroides osleri) from coyotes to domestic dogs and coyotes. Journal of Parasitology, 1981, 67, 284-286.

Hovell G.J.R. and Weston R. Cramp in greyhounds. Veterinary Record, 1970, 87, supplt 47.

Jacobs D.E. and Prole J.H.B. Helminth infections of british dogs : prevalence in racing greyhounds. Veterinary Parasitology, 1976, 1, 377-387.

Jones B.R., Clark W.T., Collins G.H. and Johnstone A.C. Filaroides osleri in a dog. New-Zealand Veterinary Journal, 1977, 25, 103-104.
Kelly P.J. and Mason P.R. Successful treatment of Filaroïdes osleri infection with oxfendazole. Veterinary Record, 1985, 116, 445-446.

Lamure-Sauvaire M.P. Intérêt de l'endoscopie dans le diagnostic des affections respiratoires chroniques chez le chien. Thèse de médecine vétérinaire, Lyon, 1992, n 62 , $120 \mathrm{p}$.

LAUder I.M. and Lawsson D.D. Differential diagnosis of some conditions of the canine and feline thorax. Veterinary Record, 1959, 71, 1096-1105.

LeCOINDRE P. and CADORÉ J.L. Manuel d'endoscopie vétérinaire. Éditions du fleuve, 1992, 220 pp.

MalHerbe W.D. The diagnosis of Filaroides osleri (Cobbold, 1879) infestation. Journal of South Africa Veterinary and Medical Association, 1951, 22, 129-130.

MalHerbe W.D. The chemotherapy of Filaroides osleri infestation in dogs : a progress report. Journal of South Africa Veterinary and Medical Association, 1954, 25, 9-12.

Milus J.H.L. Filaroidiasis in the dog : a review. Journal of Small Animal Practice, 1967, 8, 37-43.

Neumann L.G. Parasites et maladies parasitaires du chien et du chat. Vigot, 1941, 340 p.

OAKLEY G.A. and Rowlands D.T. Anthelmintics for cats and dogs. Anpar Books, 1991, 447 p.

OrtLepp R.J. The lungworm F. osleri (Cobbold, 1879) in South African bred dogs. Journal of Soutb Africa Veterinary and Medical Association, 1945, 16, 86-87.

OSLER W. Verminous bronchitis in dogs. The Veterinarian. London, 1877, 12, 387-397.

Pence D.B. and Meinzer W.P. Helminth parasitism in the coyote, Canis latrans, from the rolling plains of Texas. International Journal for Parasitology, 1979, 9, 339-344.

PILlers A.W.N. Some remarks on the clinical aspect of chronic tracheobronchitis of the dog due to Oslerus osleri (Cobbold, 1879). Veterinary Record, 1935, 15, 62-63.

POLley L. Quantitative observations on populations of the lungworm Oslerus osleri (Cobbold, 1889) in coyotes (Canis latrans Say). Canadian Journal of Zoology, 1986, 64, 2384-2386.

Polley L. and Creighton S.R. Experimental direct transmission of the lungworm Filaroides osleri in dogs. Veterinary Record, 1977, 100, 136-137.

Randolph J.F. and Rondano V.T. Treatment of Filaroides osleri infestation in a dog with thiabendazole and levamisole. Journal of American Animal Hospital Association, 1984, 20, 795-798

Urquhart G.M., Jarrett W.F.H. and O'Sullivan J.G. Canine tracheobronchitis due to infection with Filaroides osleri. Veterinary Record, 1954, 66, 143-145.

VAlet-Picavet S. Une bronchite très "vermineuse" ou filarö̈dose à Oslerus osleri chez un chien. Action Vétérinaire, $1991, \mathrm{n}^{\circ} 1157,19-22$.

Wheeldon E.B., Pirie H.M., Fisher E.W. and Lee R. Chronic respiratory disease in the dog. Journal of Small Animal Practice, 1977, 18, 229-246.

Accepté le 19 septembre 1994 